\title{
Surfaces of forced vergence disparity
}

\author{
A Rubin* \\ Department of Optometry, University of Johannesburg, PO Box 524, Auckland Park, 2006 South \\ Africa
}

<arubin@uj.ac.za>

Received 5 December 2012; revised version accepted 26 February 2013

\begin{abstract}
Purpose: To introduce the concept of surfaces of forced vergence disparity using measurements of fixation disparity from three young, healthy individuals.

Method: Fixation disparities were measured in three individuals in relation to variation in stimulus distance and vergence demand. All measurements were obtained by means of the Sheedy disparometer which can be used to measure not only fixation disparity but also associated phorias. For each individual, consecutive measurements of fixation disparity only were determined over a short time period at four stimulus distances $(0.25 \mathrm{~m}, 0.4 \mathrm{~m}$, $1 \mathrm{~m}$ and $2 \mathrm{~m}$ ) and under five different vergence demands as produced with the application of different amounts of prism (either base in or base out) and also without any prism. Parametric and non-parametric statistical methods are used to understand short-term variation of fixation disparity and pseudo-3D and stereo-pairs represent the surfaces of forced vergence disparity with which this paper is mainly concerned.
\end{abstract}

Results: Surfaces of forced vergence disparity are very useful to study variation of fixation disparity in relation to change in stimulus distance and vergence demand. They are effectively 3-dimensional equivalents of 2-dimensional Ogle curves of forced vergence disparity.

Conclusion: Surfaces of forced vergence disparity may be useful in many contexts - both in relation to normal or unusual binocular behaviour. This paper introduces such surfaces in relation to three individuals who were considered as having satisfactorybinocularandaccommodative-vergence function. The surfaces assist one in understanding complicated three-dimensional or trivariate data that involves fixation disparities, different stimulus locations and different accommodative-vergence demands upon the oculo-motor system. ( $S$ Afr Optom 2013 72(1) 25-33)

Key words: Curves of forced vergence disparity, Ogle's curves, fixation disparity, surfaces of forced vergence disparity, multivariate statistics, binocular function, accommodative-vergence dysfunction

\section{Introduction}

Fixation disparity (FD) and associated phorias (AP) are probably under-utilized clinical parameters in general optometric and ophthalmologic practice. FD is usually measured in minutes of arc and provided the value is small enough the oculo-centric (or visual) axes of the two eyes of an individual are both located within Panum's fusional area thus ensuring that fusion rather than diplopia is experienced. This paper mainly concerns fixation disparities and thus associated phorias will not be discussed in any great detail. Fixation 
disparities are sometimes confused with associated phorias but these two quantities or concepts are not the same thing. Similarly, associated and dissociated phorias (DP) are also different quantities and should not be confused. Different authors differ in terms of their understanding and definitions of fixation disparity and some $e^{1-3}$ may regard a FD as an anomaly of binocular function or vision whereas others ${ }^{4-6}$ consider small FD (and particularly smallish horizontal FD) as being a normal adaptive measure to small inter-ocular retinal disparities or differences of position of the two oculo-centric axes of the right and left eyes of an individual. In the latter approach ${ }^{4-6}$ the FD and its variation are then considered to reflect instantaneous changes in eye, and retinal image, position and FD is not generally regarded as an anomaly unless its value exceeds some particular amount (that typically ranges from 4-10 minutes of arc in the relevant literature ${ }^{4-8}$ ) and where symptoms of binocular discomfort are present. Vertical FD, on the other hand, is typically considered as being more of a problem and a forced vertical fixation disparity (FVFD) function can be measured using small increments of prism (base up or down before one eye). Usually, but not always, a straight line is found when the FVFD function is plotted and where the slope is steep, that is within $45^{\circ}$ of the vertical axis (assuming right hyper FD and left hyper FD with $\mathrm{BU}$ and $\mathrm{BD}$ prism before OD) then this has been considered as a sign of inadequate vertical prism adaptation ${ }^{9}$. Some patients, however, show a curve for the FVFD function, not unlike a Type I Ogle curve ${ }^{9}$. However, this paper will not focus any further on vertical FD and will emphasize horizontal FD and its short-term variation in relation to change in two specific variables, namely stimulus distance and vergence demand as induced through the use of prism. Treatment, whether in the form of vision therapy, lenses or prisms, or even occasionally extra-ocular muscle surgery or medications will naturally differ according to the clinical meaning and relevance that different clinicians and others may ascribe to FD and so the issue of properly understanding the real significance of FD is not a simple or trivial one.

Both subjective , $^{7-10}$ and objective methods s, $^{7-10}$ can be used to determine FD and this paper will use a subjective approach (the Sheedy disparometer ${ }^{1,7}$ ), as it is perhaps one of the more commonly applied clinical methods. Examples of objective methods ${ }^{10}$ include the use of eye movement trackers via infrared sensors or scleral coils embedded within contact lenses that are worn by the subject or patient. Besides the Sheedy disparometer, there are also a range of other subjective methods such as the Mallett box and Woolf, Wesson or Saladin cards ${ }^{11-15}$. Many of the subjective methods use polarizers and nonius lines to measure FD or associated phorias. In some parts of the world, such as Europe, a sensory approach (rather than a motor one as in curves of forced vergence disparity) to measurement and analysis of fixation disparity gradually developed $^{16}$, wherein FD was considered as a sensory adaptation to small errors of ocular alignment.

In 1949, Ogle proposed clinical measurement of fixation disparity and later forced vergence disparity curves $^{5,16}$ although Hofmann and Bielschowsky had already used Hering's haploscope in about 1900 to perform various experiments involving binocular fusion. In Figure 1 below a typical type I Ogle curve is provided to assist with a brief explanation of the relevant quantities of clinical interest such as the slope and $x$ - and $y$-intercepts (see the figure caption for a brief explanation of the meaning of these quantities). The slope of the curve varies (depending on vergence demand) but in Figure 1 we can see that the slope remains similar (the flat portion in the middle of the curve intersecting with the dashed line). Generally, clinically the slope can be determined by measuring FD for three vergence demands, usually 5 pd BI, 0 and 5 pd BO. Some authors ${ }^{9}$ prefer to measure the slope between 3 pd BI and 3 pd BO instead. Such measurements can be done quite simply and quickly using a Sheedy disparometer or alternative method at, say, $0.4 \mathrm{~m}$. Clinically the slope is sometimes regarded as being the parameter with the closest correlation to the presence of symptoms ${ }^{17,18}$ and changes in the slope are measured where treatments such as vision therapy might be attempted. A steep or absolute slope of $>1$ min arc per prism dioptre is considered as being potentially problematic ${ }^{16}$, 19. A simple example is included here to assist in understanding and application. Example 1: Suppose the FD was 11 min arc as determined through 3 pd BI and 9 min arc through 3 pd BO then the slope would be: 
slope $=\frac{y_{2}-y_{1}}{x_{2}-x_{1}}=\frac{9-11 \mathrm{~min} \text { arc }}{3-(-3) \mathrm{pd}}$

$\approx-0.33 \min \operatorname{arc} / \mathrm{pd}$

$\mid$ slope $\mid=0.33 \mathrm{~min}$ arc $/ \mathrm{pd}$

Note that the $\mathrm{BI}$ value of 3 is given a negative sign to differentiate it from the $3 \mathrm{BO}$ value and avoid problems with equation 1 where it would otherwise be undefined. Here the modulus or absolute slope (|slope|) was not greater than $1 \mathrm{~min} \mathrm{arc} / \mathrm{pd}$ and so we would not expect the patient to have any problems with activities such as reading or other proximal or near activities, or at least in so far as slopes and FD are concerned. (Some authors would consider FD of 9 or 11 min arc as being relatively large and on the limit of the normal range of values.) Of course, there could be other factors that could possibly create difficulties with proximal activities in certain persons other than FD-related ones.

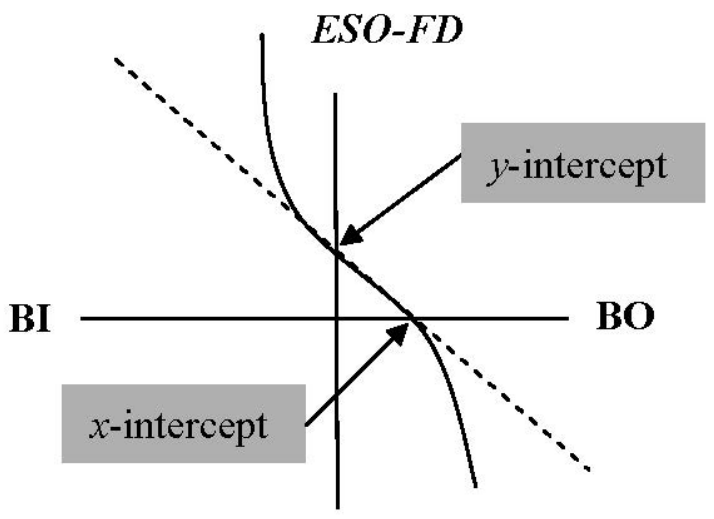

$E X O-F D$

Figure 1. An example of a typical Type I Ogle curve of forced vergence disparity is indicated. The $x$-axis or axis of forced or induced vergence demand, has units of prism dioptres and can range from say $20 \mathrm{pd} \mathrm{BI}$ to $30 \mathrm{pd} \mathrm{BO}$, depending on stimulus distance, measurement method and other issues. The $y$-axis, or axis of $F D$, has units of minutes of arc and positive values indicate eso-FD. The intersect of the two axes or origin would represent 0 pd FD with no prism before the two eyes. As BO prism is increased in magnitude, so greater convergence is induced; while increasing BI values indicates greater forced divergence. The curve (of forced vergence disparity) indicates the unique fixation disparities that this hypothetical subject displayed through the corresponding induced vergence posture. The $y$-intercept indicates the FD that this individual had through $0 \mathrm{pd}$ while the $x$-intercept is the associated phoria, that is, the amount of prism (here a $\mathrm{BO}$ value of some sort) that was needed to reduce the FD to zero.
The primary purpose of this paper is to introduce the concept of surfaces of forced vergence disparity (see Figure 4a for an example) using clinical data measured from three, young, healthy subjects. Such a surface for an individual is a three-dimensional representation of the complicated trivariate relation between three quantities, namely FD, vergence demand and stimulus distance (which, of course, also reflects accommodative demand).

\section{Method}

The study proposal satisfied the requirements of the Declaration of Helsinki and was approved according to the required process of the University of Johannesburg. After signing the applicable informed consent document, each subject also completed a simple, mainly biographical questionnaire that also included some questions on general and ocular health relevant to the purposes of this study. Various standard procedures such as retinoscopy, subjective refraction, stereo-acuity thresholds and cover test were performed and thereafter a Sheedy Disparometer and polarised filters were used to repeatedly measure fixation disparities for two female and one male subject, all 21 or 22 years of age and nearly emmetropic. All subjects reported that they were healthy and free of binocular problems or related symptoms. All FD measurements were obtained with the use of trial frames and with larger amounts of prism distributed between the two eyes (mainly to reduce the possibility of slight distortions of vision with, say, a single larger prism of 15 or $20 \mathrm{pd}$ ). Ambient luminance was similar for all three subjects and measurements were obtained under photopic conditions. (Measurements with an anaglyphbased and computerized fixation disparity procedure were also obtained for one of the three subjects but they will not be included in the analysis here.)

Fixation disparities were measured at four different stimulus distances, namely $0.25,0.5,1$ and 2 metres and at each of these distances 10 measurements were obtained through the appropriate binocular refractive compensation (the three participants were selected specifically as they were all almost emmetropic). Vergence demands were changed using prism and all subjects had measurements performed with no prism and also with base-in (BI) and base-out (BO) prism ranging from $15 \mathrm{pd} \mathrm{BI}$ to $20 \mathrm{pd} \mathrm{BO}$. An attempt was made with all subjects to use the same amounts of 
prism to create similar vergence demands but this was not possible with the base-in or divergence-inducing prisms. In all subjects, at the closest stimulus distances $(0.25$ and $0.5 \mathrm{~m})$ fixation disparity measurements were obtained with $15 \mathrm{pd} \mathrm{BI,} 10 \mathrm{pd} \mathrm{BI,} 0 \mathrm{pd}, 10 \mathrm{pd} \mathrm{BO}$ and $20 \mathrm{pd} \mathrm{BO}$ but this was not true for the further stimulus distances such as 1 or $2 \mathrm{~m}$ where difficulties with divergence in all of the three subjects required the $\mathrm{BI}$ or divergence demand and prisms to be reduced in value to allow fusion to occur.

For each participant a total of 200 measurements of fixation disparity were obtained for the four stimulus distances and five different vergence demands per distance. This whole measurement process required about 45-60 minutes per individual and short rest periods were provided as necessary between measurements.

Mydriatics and cycloplegics were not used in this study and thus binocular and accommodative functions were preserved.

\section{Statistical analysis}

Fixation disparities for the three subjects were analysed using Statistica and Matlab. Results are shown using both bivariate and multivariate methods of graphical representation such as box-and-whisker plots, pseudo-3D surfaces and stereo-pairs.

\section{Results}

Results are shown for Subject 1 (in Figure 2a) for a stimulus distance of $0.25 \mathrm{~m}$, or an accommodative demand of $4 \mathrm{D}$. The $x$-axis represents vergence demand in prism dioptres (pd) while the $y$-axis indicates fixation disparity in min arc. (See Figure 1 where the two axes are essentially identical although no values or numbers have been included.) In Figure 2 a five box-and-whisker plots and a single red curve are shown passing through the sample means (the five small squares) for the samples of fixation disparities for the five conditions or vergence demands, namely $15 \mathrm{pd}$ base in (15 BI), $10 \mathrm{BI}, 0,10$ base out (10 BO) and $20 \mathrm{BO}$ evaluated. This red curve is comparable to an Ogle type forced vergence disparity curve and here with base in (BI) vergence demand, that is, a demand for divergence the curve is similar to a Type 1 Ogle curve but with convergence (or BO prism) the curve is relatively flat with means near zero FD. Larger boxes represent the respective means plus and minus one standard deviation for the respective samples measured. (The whiskers similarly indicate the applicable sample mean plus and minus 1.96 standard deviations.) For a vergence demand of zero, we note that the mean was 4.4 exo-FD (actually -4.4 on the axis) and the standard deviation (9.9 min arc) was the largest, that is, the rectangle is the biggest whereas the fixation disparities were much less variable when measured, for example, through a vergence demand of $10 \mathrm{BI}$.

For the same subject in Figure 2b instead, sets of five box-and-whisker plots each for (four) stimulus distances are included. The box-and-whisker plots within the black ellipse are the same as in Figure 2a. Similar results for the other three stimulus distances $(50 \mathrm{~cm}, 1$ and $2 \mathrm{~m})$ are also included and the red curves indicate mainly Type 1 Ogle curves. This figure, although not the simplest, includes quite a lot of information about mean fixation disparities and their variation at different distances and under different vergence demands.

Figure 3 illustrates the same data as in Figure 2a but in a pseudo 3D-space. The red curve is basically an Ogle type curve of forced vergence disparity but plotted in a 3-dimensional pseudospace. The axes are now not only vergence demand (in pd) and FD (in min $a r c$ ) but an additional axis indicates the stimulus distance (in metres) that is, the accommodative stimulus or demand. (We could, of course, have plotted all the data in Figure $2 \mathrm{~b}$ within Figure 3 but to simplify matters only results for one stimulus distance was shown.)

a)

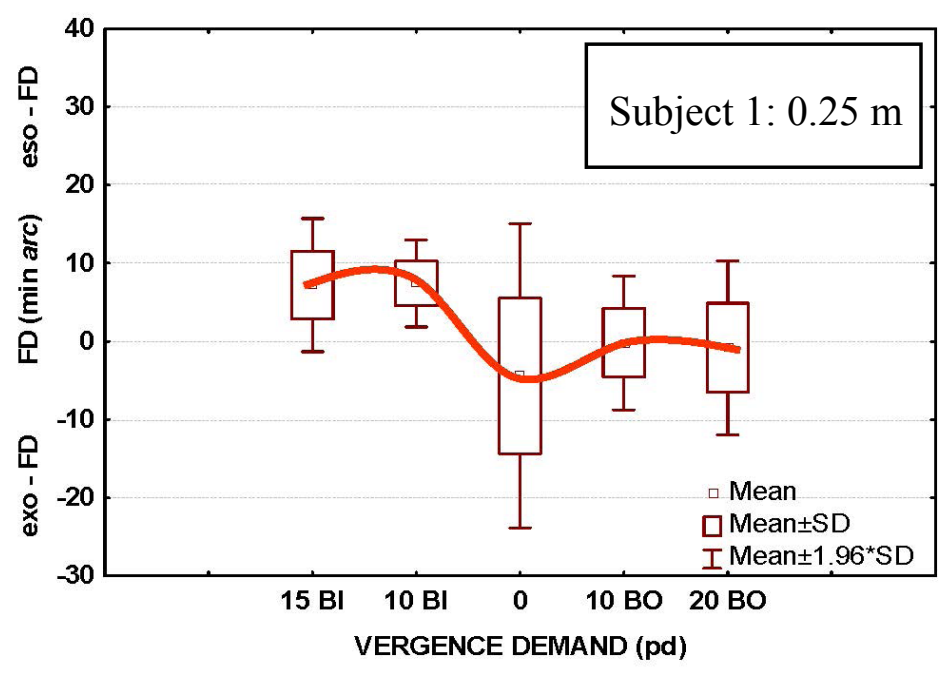


b)

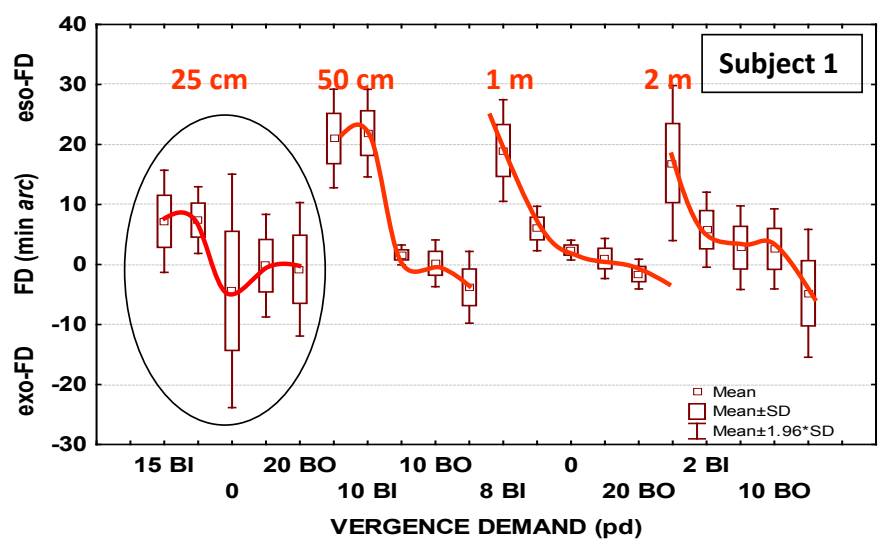

Figure 2. Box and whisker plots for Subject 1 for the different vergence demands and for different stimulus distances are shown. In part a) only results for one stimulus distance $(0.25 \mathrm{~m})$ are included whereas in part b) these same results and also those for three other stimulus distances are included. Small squares with red curves indicate mean fixation disparities (FD) under specific vergence demands (measured with no prism, or with base in or base out prism.) Larger boxes and whiskers are used to indicate sample variation by means of sample standard deviations. a) Subject $1,0.25 \mathrm{~m}$, b) Subject 1, 0.25, 0.5, 1 and $2 \mathrm{~m}$.

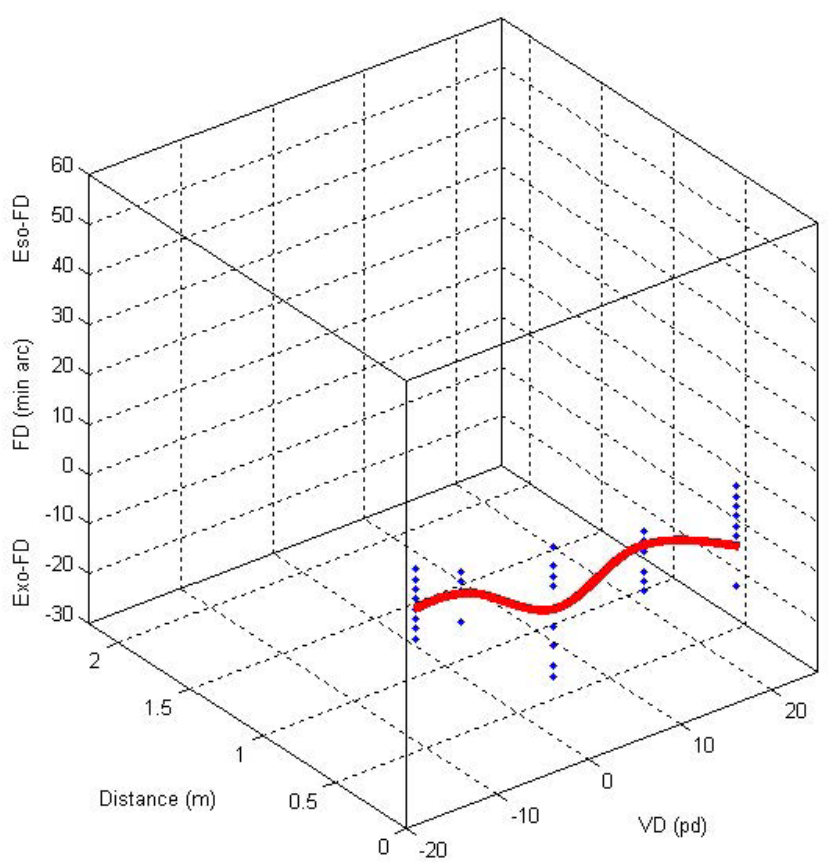

Figure 3. The same data, as in Figure 2a for Subject 1, is included to illustrate the transition from a 2 -space to a 3-space. Here only results for $0.25 \mathrm{~m}$ are shown but one could easily include similar data for other stimulus distances such as $0.5,1$ and $2 \mathrm{~m}$ as for Figure $2 \mathrm{~b}$.
In Table 1 sample means and standard deviations for the three subjects under five different vergence demands and four stimulus distances are indicated. For Subject 1, these means and standard deviations are also indicated in Figures $2 \mathrm{a}$ and $\mathrm{b}$.

In Figure 4, results for each of the three subjects are indicated in the same 3-space as used in Figure 3. For example, Figure 4a indicates all measurements for Subject 1, that is, the raw data or fixation disparities (blue dots) measured at the four stimulus distances $(0.25,0.5,1$ and $2 \mathrm{~m}$ ) and under the five different vergence demands used. A best-fit surface of forced vergence demand is fitted to the measured data for the subject and warmer and colder colours respectively indicate either eso-FD or exo-FD. The colour bar on the right of the surface (in Figure 4a, for instance) indicates the FD in min arc and red would indicate eso-FD while dark or navy blue indicates exo-FD. All three subjects indicate increased eso-FD under forced divergence (through the addition of base in prism) irrespective of the stimulus distance applicable. That is, with forced divergence the surface changes from blue to warmer colours (red, orange or yellow) and the surfaces themselves are irregular or not flat. On the other hand, with forced convergence (the addition of base out prism) the surfaces for each of these three subjects remain relatively flat and blue or dark blue irrespective of stimulus distance.

In Figures 5a and b, the surface for Subject 1 (as in Figure $4 \mathrm{a}$ also) is simply rotated so that the axis for FD is perpendicular to the plane of the page and thus the colour (and changes or variation in FD) with respect to variation in both stimulus distance and vergence demand are clearly indicated. Figures $5 \mathrm{a}$ and $\mathrm{b}$ are essentially two different graphical or analytical methods for representing the same data (but the surface is calculated using different fitting methods and thus is similar but not exactly the same in the two parts of Figure 5). These figures are included to show that one can represent such surfaces using different graphical and mathematical approaches and some of these methods may be perhaps easier or more difficult to understand but sometimes the use of more than one methods may be useful to see various aspects of the surface and data that might otherwise be less obvious. 
Table 1. Means and standard deviations for fixation disparities (FD) measured for three young subjects at four different stimulus distances $(0.25,0.5,1$ and $2 \mathrm{~m}$ ) under different vergence demands induced with Base In (BI) or Base Out (BO) prism. In some instances, the subject concerned had difficulty fusing under the vergence demand and prism had to be reduced - this was noted for the greater stimulus distances such as 1 or $2 \mathrm{~m}$ and with demand on divergence (using BI prism) rather than convergence (with BO prism). The units are min arc throughout the table and positive and negative values refer to eso-FD and exo-FD respectively. All means are for samples of ten $(N=10)$ measurements each obtained for the specified vergence demand and stimulus distance.

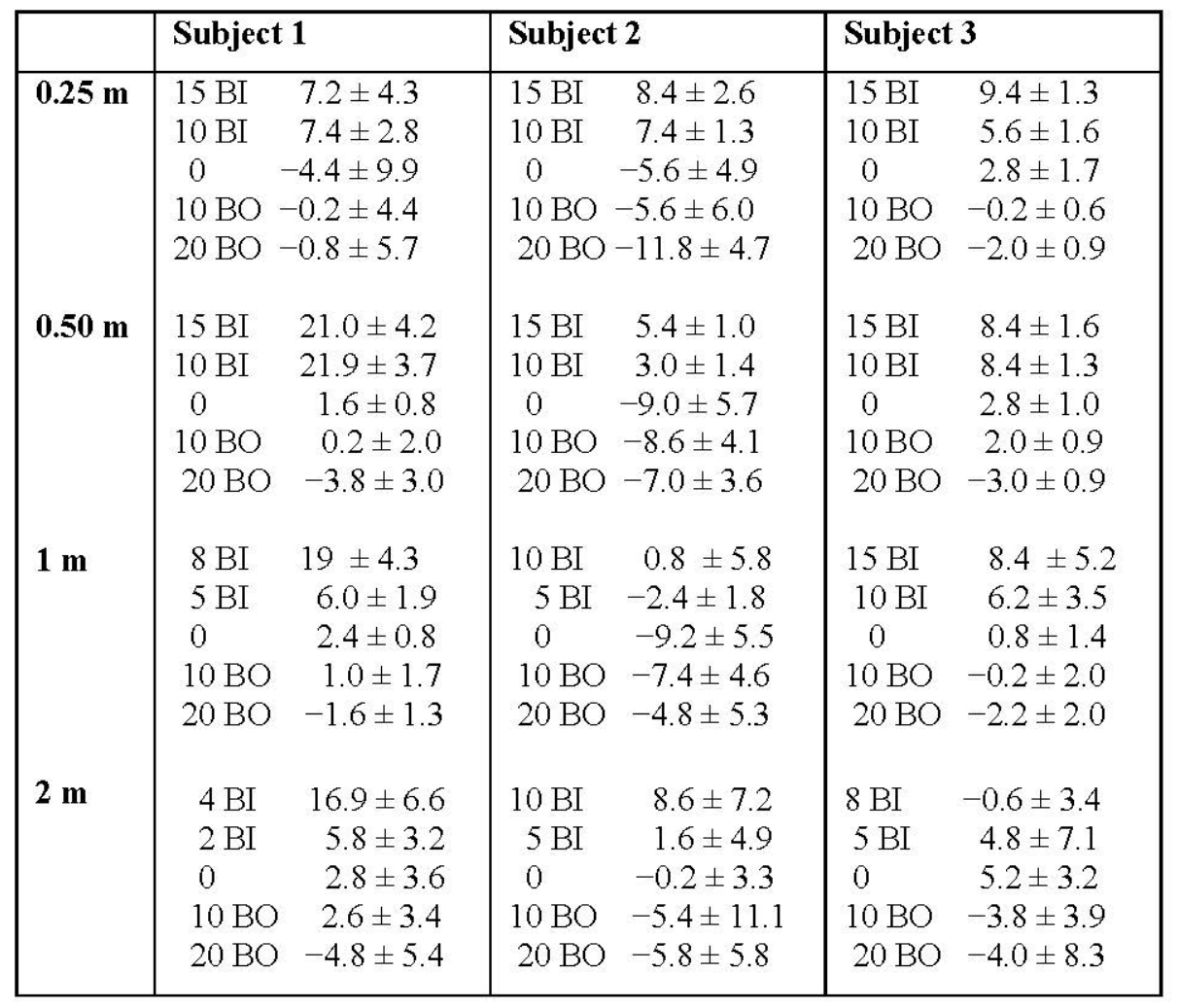

b)

a)
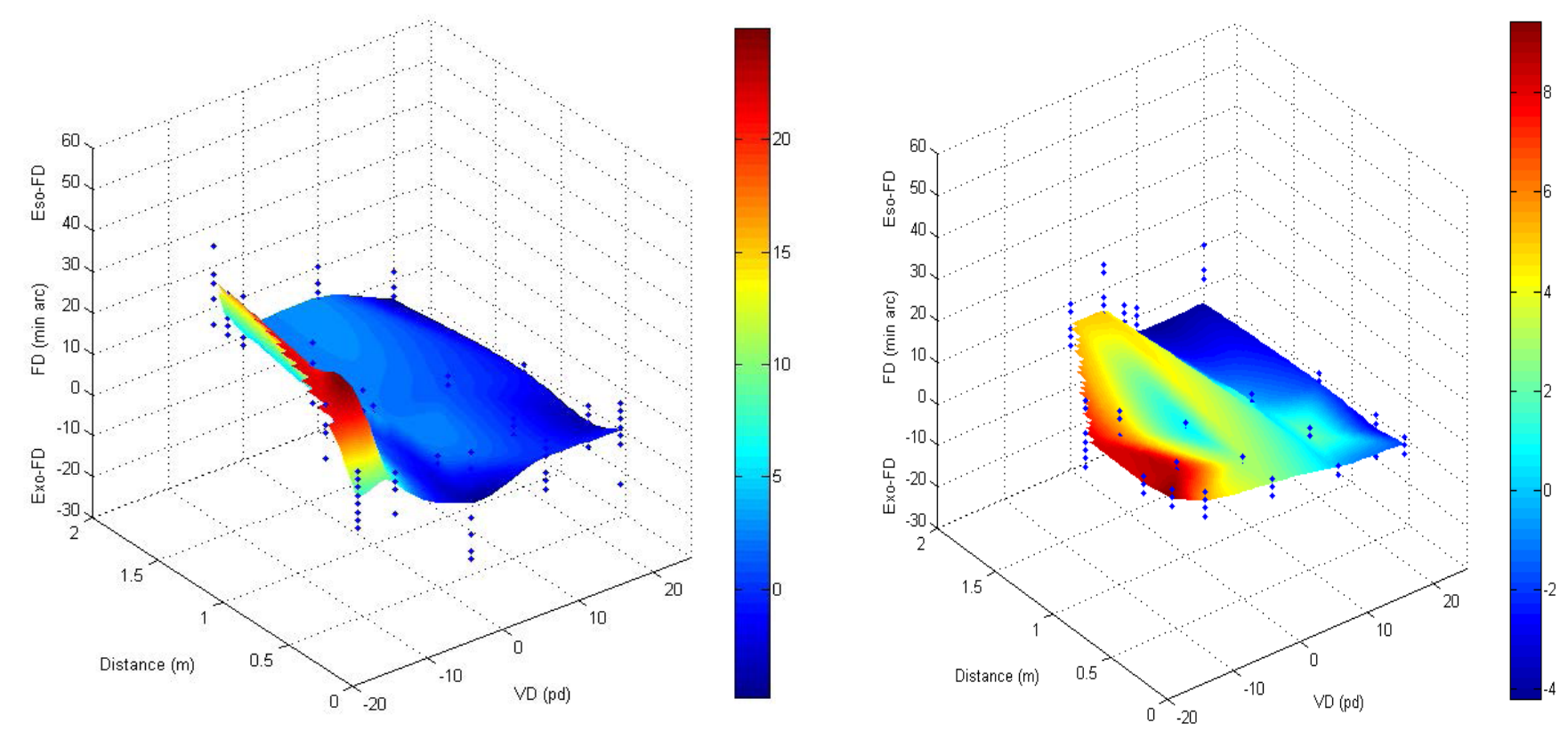
c)

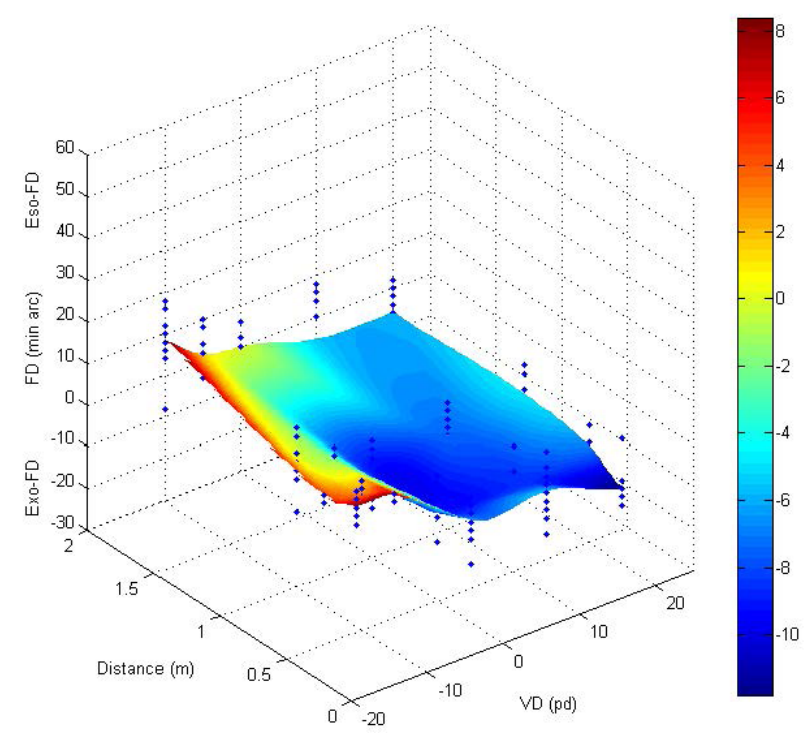

Figure 4. Pseudo-3D plots for surfaces of forced vergence disparity for three subjects. a) Subject 1, b) Subject 2 and c) Subject 3. The colour bar on the right side represents fixation disparity in $\min$ arc.

a)

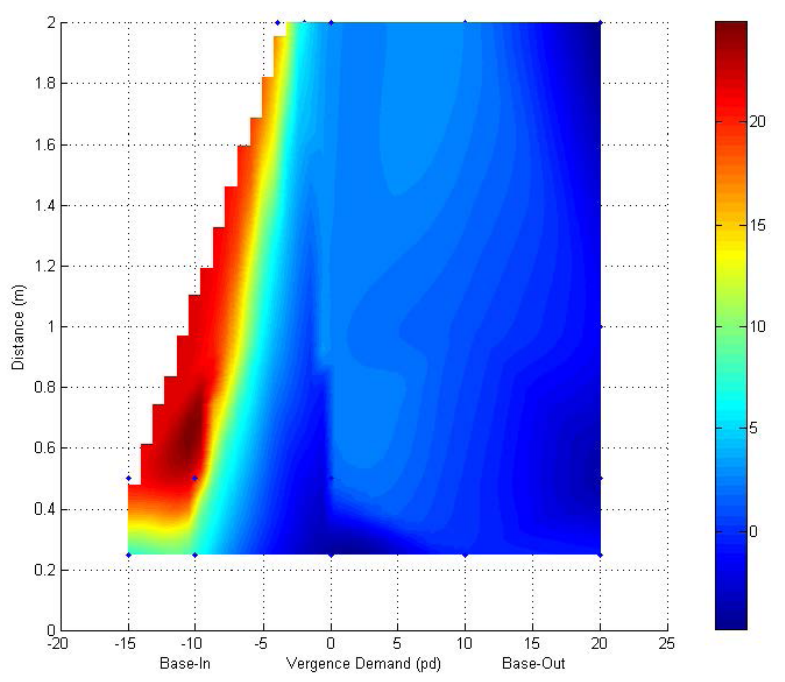

b)

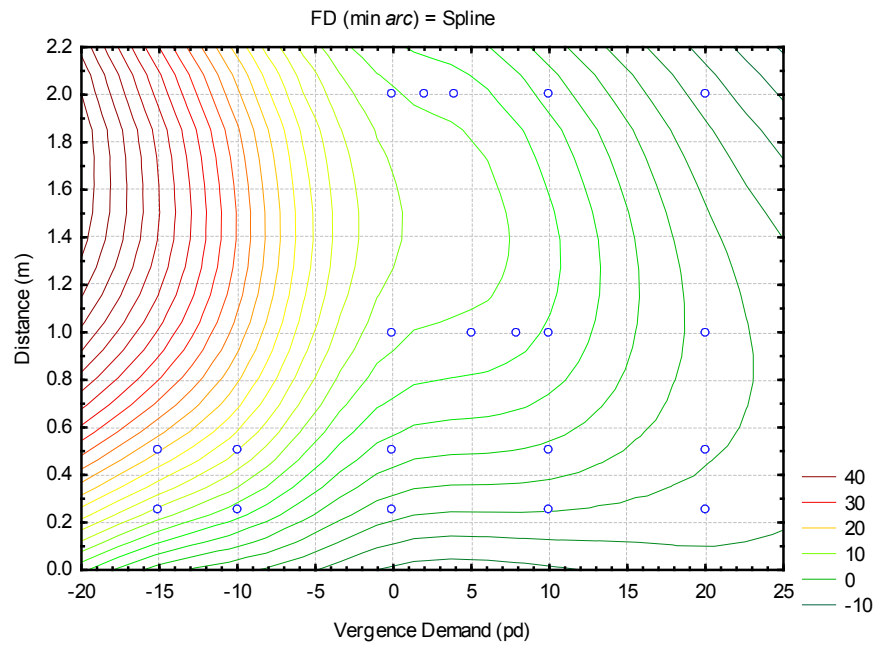

Figure 5. Surfaces of forced vergence disparity for Subject 1 illustrated with different fitting methods in parts a and b. Part $\mathbf{a}$ is merely a different view of Figure $4 \mathrm{a}$ whereas in Figure $5 \mathrm{~b}$ curves of iso-FD in the surface of forced vergence disparity are included and their colours indicate different values for FD. Warmer colours (red or orange) indicate iso-curves of larger amounts of eso-FD while colder colours (such as bright green, green or dark green indicate iso-curves for $10 \mathrm{pd}$ eso-FD, zero or $0 \mathrm{pd}$ FD or $10 \mathrm{pd}$ of exo-FD respectively.

In Figure 6 a stereo-pair of the surface of forced vergence disparity for Subject 1 is shown. (This is the same surface as shown in Figure 4a.) Readers should allow their eyes to drift (into an exo-posture) so that they are fixating at an imaginary point behind the plane of the paper (or instead one can fixate at an arbitrary point somewhere in front of the figure).

\section{Discussion and Conclusion}

In contrast to the more commonly known curves of forced vergence disparity ${ }^{5}$ (see Figure 1) from Ogle, this paper introduces and illustrates in 3-dimensional pseudospace (Figures 4 and 5) and also in a stereopair (Figure 6) the idea of surfaces of forced vergence disparity (SFVD). Such surfaces allow one to more easily consider and understand variation in fixation disparity in relation to variation or change in both stimulus distance and vergence demand as induced using ophthalmic prisms. In some sense the surfaces tell us about the interactions and interrelations between accommodation (related to stimulus position or distance), vergence and ocular fusional behaviour. They thus summarize or describe a complicated interaction of human vision and ocular function or behaviour and could potentially be important in both diagnosis and management of binocular anomalies. Variation in such surfaces in relation to vision therapy could, for example, be an interesting area for future investigation. Other areas for further research would be age-related effects on such surfaces, diurnal variation in SFVD, and changes of the surfaces in relation to conditions that may influence ocular musculature such as myasthenia gravis, thyroid disease, leukemia or diabetes.

In this paper results from only three young subjects were used for the illustration of SFVD. The instrument used to measure the fixation disparities was the Sheedy disparometer; an instrument that can be easily used in free space with auxiliary prism, either base in or base out. At all stimulus distances investigated and under all vergence demands small samples of ten measurements (that is, fixation disparities) each were determined and 

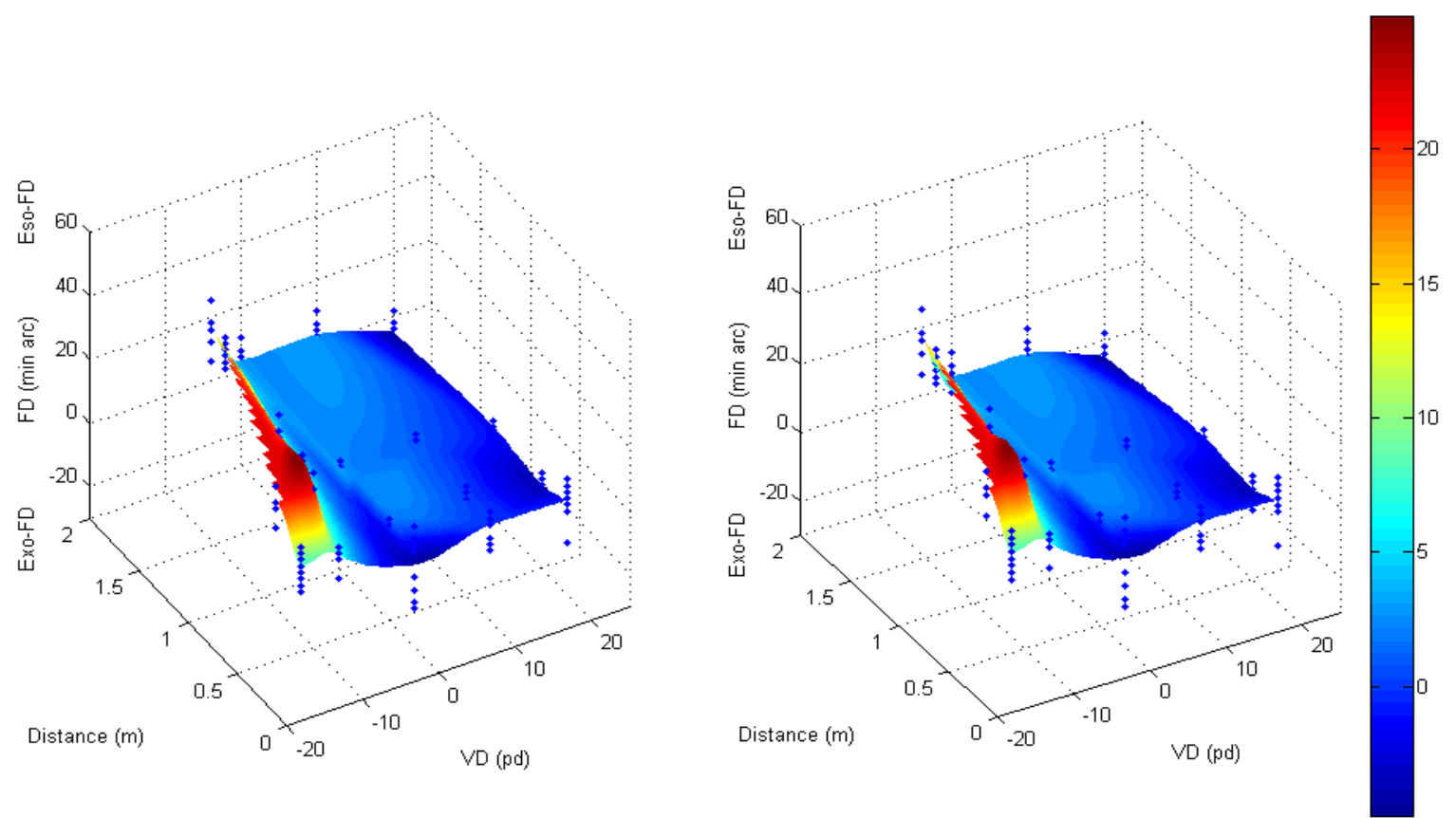

Figure 6. Stereo-pair plot of the surface of forced vergence disparity for Subject 1. Warmer colours (red and orange) represent larger eso-FD with BI prism or forced divergence at the various distances concerned. The blue dots are the raw measurements to which this best-fit surface has been fitted. The colour bar on the right side represents fixation disparity in min arc.

then means and other descriptive statistics (see Table 1) were calculated for further analysis and graphical representation. Collectively, 200 measurements were obtained per subject and thus the process was fairly time-consuming and subjects were inclined to fatigue despite short rest periods being allowed. But, the primary purpose of this paper was more to introduce the idea of SFVD rather than to worry too greatly with the actual values for the fixation disparities themselves. Thus the possibility exists that the measurements might be a little more variable than otherwise due to the nature of the experimental instrument used and other factors such as possible fatigue or boredom of participants in the study. These factors, while obviously important, were nonetheless not regarded as critical issues here and further studies can be designed to more closely control some of these issues, despite them being somewhat difficult to manage in any event. Similarly, the sample means themselves may have been affected by the same issues.

The surfaces could be determined using other subjective or objective methods ${ }^{10}$ for measurements of FD such as Wesson ${ }^{11,12-13}$ or Saladin ${ }^{12-13}$ cards. Computerized methods ${ }^{6}$ can also be used to measure FD and one such method, developed by the author, was also used in one of the three subjects of this paper but for the sake of simplicity this data was not included here. But, the advantage of such computerized methods is that multiple measurements of FD can be obtained more quickly and thus experimental concerns such as participant fatigue or boredom become less of an issue.

Obtaining the necessary measurements here for a surface of forced vergence disparity required about 45 to 60 minutes per subject. But, for the purposes of this research, sets of 10 measurements per stimulus distance and per vergence demand necessitated 200 measurements per subject or per surface. Clinically, it would not be necessary to obtain so many measurements and one might simply measure one measurement per vergence demand and per stimulus distance. One might also reduce the number of stimulus distances and also vergence demands used, and so the time necessary to obtain the measurements could be dramatically reduced. In a clinical situation it would be quite feasible to get the required measurements for a patient in less than one minute and this would especially be true with a computerized approach ${ }^{6}$ to measurement of FD, instead of using the Sheedy disparometer ${ }^{11,13}$. The SFVD could have diagnostic importance and also could be utilized as a clinical method for monitoring change in a patient in relation to various factors such as ageing, disease or with medical treatment. Changes in such surfaces could also be used to study the possible effects 
of vision therapy or other treatments such as prisms, lenses or surgery for oculomotor or accommodativevergence dysfunction.

\section{Acknowledgements}

Y Rosin, S Joubert, T Langa, H Shamase, S Chauke and S Naransamy are thanked for their important assistance and contributions towards measurement and the initial analysis of the raw data of the subjects that are the basis for this paper. Thanks are also extended to the three subjects who participated in this study.

\section{References}

1. Sheedy JE, Saladin JJ. Phoria, vergence and fixation disparity in oculomotor problems. Am J Optom 1977 54(7) 474-478.

2. Calaroso EE, Rouse MW. Clinical management of strabismus. Boston: Butterworth-Heinemann, 1993, pp.105-108.

3. Mallett RFJ. Fixation disparity - its genesis in relation to asthenopia. Ophthal Physiol Opt 197414 1159-1168.

4. Schor CM, Neuromuscular plasticity and rehabilitation of the ocular near response. Optom Vis Sci 200986 788-802.

5. Ogle KN, TGMartens, JADyer. Oculomotor imbalance in binocular vision and fixation disparity. London: Henry Kimpton, 1967.

6. Jaschinski W, Kloke W, Jainta S, Buchholz J. Horizontal fixation disparity measures with nonius lines. Optom Vis Sci 200582988 999.

7. Benjamin WJ (editor). Borish's clinical refraction. Philadelphia: Saunders, 1998, pp.739-742, 752-754, 759-765.

8. Ukwade MT. effects of nonius line and fusion lock parameters on fixation disparity. Optom Vis Sci 200077 309-320.

9. Despotidis N, Petito GT. Fixation disparity: clinical implications and utilization. J Am Optom Assoc 199162 923-932.

10. Fogt N, Jones R. Comparison of fixation disparities obtained by objective and subjective methods. Vis Res 199838 411421.

11. Goss DA, Patel J. Comparison of fixation disparity curve variables measured with the Sheedy disparometer and the Wesson Fixation Disparity Card. Opt Vis Sci 199572 580588 .

12. Ngan J, Goss DA, Despirito J. Comparison of fixation disparity curve parameters obtained with the Wesson and Saladin fixation disparity cards. Optom Vis Sci 200582 6974.

13. Frantz KA, Elston P, Michalik E, Templeman CD, Zoltoski RK. Comparison of fixation disparity measured by Saladin card and disparometer. Optom Vis Sci 201188 E733-E741.

14. Corbett, A, Maples WC. Test-retest reliability of the Saladin card. Optom 200475 629-639.

15. Griffin JR. Binocular anomalies for vision therapy, $2^{\text {nd }}$ ed. New York: Professional Press Books/ Fairchild Publications, 1982.

16. London R, Creleir RS. Fixation disparity analysis: sensory and motor approaches. Optom 200677 590-608.
17. Schor CM. The influence of rapid prism adaptation upon fixation disparity. Vis Res 197919 757-765.

18. Sheedy JE, Saladin JJ. Association of symptoms with measures of oculomotor deficiencies. Am J Optom Physiol Opt 197855 670-676.

19. Irving EL, Robertson KM. Monocular components of the fixation disparity curve. Opt Vis Sci 199168 117-126. 